\title{
New generalizations of Darbo's fixed point theorem
}

\author{
Longsheng Cai and Jin Liang*
}

*Correspondence: jinliang@sjtu.edu.cn

Department of Mathematics, Shanghai Jiao Tong University, Shanghai, 200240, P.R. China

\begin{abstract}
In this paper, we propose a new idea to investigate the important basic problem: how to extend Darbo's fixed point theorem? We establish some new generalizations of Darbo's fixed point theorem by using 'integral conditions'. Our fixed point theorems extend the existing results on the problem above.
\end{abstract}

\section{Introduction}

It is well known that the Schauder fixed point theorem plays an important role in nonlinear analysis. In 1955, Darbo [1] proved a fixed point property for $\alpha$-set contraction on a closed, bounded and convex subset of Banach spaces in terms of the measure of noncompactness, which was first defined by Kuratowski [2]. Darbo's fixed point theorem is a significant extension of the Schauder fixed point theorem, and it also plays a key role in nonlinear analysis especially in proving the existence of solutions for a lot of classes of nonlinear equations. Since then, some generalizations of Darbo's fixed point theorem have appeared. For example, we refer the reader to [3-6] and the references therein.

In this paper, we propose a new idea to investigate the important basic problem: how to extend Darbo's fixed point theorem? We establish some new generalizations of Darbo's fixed point theorem by using 'integral conditions'. Our fixed point theorems extend the existing results on the problem above.

First of all, let us recall some basic concepts, notations and known results which will be used in the sequel. In this paper, we let $E$ be a Banach space with the norm $\|\cdot\|$ and 0 be the zero element in $E$. The closed ball centered at $x$ with radius $r$ is denoted by $B(x, r)$, by simply $B_{r}$ if $x=0$. If $X$ is a nonempty subset of $E$, then we denote by $\bar{X}$ and $\operatorname{co}(X)$ the closure and closed convex hull of $X$, respectively. Moreover, let $\mathfrak{M}_{E}$ be the family of all nonempty compact subsets of $E$.

We use the following definition of the measure of noncompactness, which is in the form of an axiomatic way.

Definition 1.1 A mapping $\mu: \mathfrak{M}_{E} \rightarrow R_{+}=[0,+\infty)$ is said to be a measure of noncompactness in $E$ if it satisfies the following conditions:

(1) the subfamily

$$
\operatorname{ker} \mu=\left\{X \in \mathfrak{M}_{E}: \mu(X)=0\right\}
$$

is nonempty and $\operatorname{ker} \mu \subset \mathfrak{M}_{E}$;

(c) 2015 Cai and Liang. This article is distributed under the terms of the Creative Commons Attribution 4.0 International License (http://creativecommons.org/licenses/by/4.0/), which permits unrestricted use, distribution, and reproduction in any medium, provided you give appropriate credit to the original author(s) and the source, provide a link to the Creative Commons license, and indicate if changes were made. 
(2) if $X \subset Y$, then $\mu(X) \leq \mu(Y)$;

(3) $\mu(\bar{X})=\mu(X)$;

(4) $\mu(\operatorname{co}(X))=\mu(X)$;

(5) $\mu(\lambda X+(1-\lambda) Y) \leq \lambda \mu(X)+(1-\lambda) \mu(Y)$ for $\lambda \in[0,1]$;

(6) if $\left\{X_{n}\right\}$ is a nested sequence of closed sets in $\mathfrak{M}_{E}$ and $\lim _{n \rightarrow \infty} \mu\left(X_{n}\right)=0$, then the intersection set $X_{\infty}=\bigcap_{n=1}^{\infty} X_{n}$ is nonempty.

The subfamily ker $\mu$ defined in (1) represents the kernel of the measure $\mu$ of noncompactness and since

$$
\mu\left(X_{\infty}\right)=\mu\left(\bigcap_{n=1}^{\infty} X_{n}\right) \leq \mu\left(X_{n}\right),
$$

we see that

$$
\mu\left(\bigcap_{n=1}^{\infty} X_{n}\right)=0 .
$$

Therefore, $X_{\infty} \in \operatorname{ker} \mu$.

Definition 1.2 Let $\Psi, \Phi:[0,+\infty) \rightarrow R$ be two functions. The pair of functions $(\Psi, \Phi)$ is said to be a pair of shifting distance functions if the following conditions hold.

(1) For $u, v \in[0,+\infty)$ if $\Psi(u) \leq \Phi(v)$, then $u \leq v$.

(2) For $u_{n}, v_{n} \in[0,+\infty)$ with

$$
\lim _{n \rightarrow \infty} u_{n}=\lim _{n \rightarrow \infty} v_{n}=w,
$$

if $\Psi\left(u_{n}\right) \leq \Phi\left(v_{n}\right)$ for all $n$, then $w=0$.

Example 1.3 Take

$$
\Psi(t)=\frac{-1+2 t}{2}, \quad \Phi(t)=\frac{-1+t}{2} .
$$

Then it is clear that they are a pair of shifting distance functions.

Theorem 1.4 (Schauder's fixed point theorem) Let $\Omega$ be a nonempty, bounded, closed and convex subset of a Banach space E. Then each continuous and compact map $F: \Omega \rightarrow \Omega$ has at least one fixed point in $\Omega$.

As a generalization of the Schauder fixed point theorem, we have the following fixed point theorem.

Theorem 1.5 (Darbo's fixed point theorem) Let $\Omega$ be a nonempty, bounded, closed and convex subset of a Banach space $E$ and let $T: \Omega \rightarrow \Omega$ be a continuous mapping. Assume that there exists a constant $k \in[0,1)$ such that

$$
\mu(T X) \leq k \mu(X)
$$


for any nonempty subset $X$ of $\Omega$, where $\mu$ is a measure of noncompactness defined in $E$. Then $T$ has at least one fixed point in $\Omega$.

\section{Main results}

In this section, we present and prove our new generalizations of Darbo's fixed point theorem.

Theorem 2.1 Let $\Omega$ be a nonempty, closed, bounded and convex subset of a Banach space $E$ and let $T: \Omega \rightarrow \Omega$ be a continuous mapping which satisfies the following inequality:

$$
\Psi\left(\int_{0}^{\mu(T X)} \varphi(t) d t\right) \leq \Phi\left(\int_{0}^{\mu(X)} \varphi(t) d t\right)
$$

for any nonempty subset $X$ of $\Omega$, where $\mu$ is an arbitrary measure of noncompactness and $\Psi, \Phi:[0,+\infty) \rightarrow R$ are a pair of shifting distance functions. Moreover, let $\varphi:[0,+\infty) \rightarrow$ $[0,+\infty]$ be a Lebesgue-integrable function, which is summable on each compact subset of $[0,+\infty)$ and

$$
\int_{0}^{\varepsilon} \varphi(t) d t>0 \text { for each } \varepsilon>0 .
$$

Then $T$ has at least one fixed point in $\Omega$.

Proof Define a sequence $\left\{\Omega_{n}\right\}$ as follows:

$$
\Omega_{0}=\Omega \quad \text { and } \quad \Omega_{n}=\operatorname{co}\left(T \Omega_{n-1}\right), \quad n \geq 1 .
$$

If there exists an integer $N \geq 0$ such that $\mu\left(\Omega_{N}\right)=0$, then $\Omega_{N}$ is compact and since $T\left(\Omega_{N}\right) \subseteq \Omega_{N}$. Thus Theorem 1.4 implies that $T$ has a fixed point.

Next, we suppose that

$$
\mu\left(\Omega_{n}\right)>0 \quad \text { for all } n \geq 0 .
$$

Then

$$
\begin{aligned}
\Psi\left(\int_{0}^{\mu\left(\Omega_{n+1}\right)} \varphi(t) d t\right) & =\Psi\left(\int_{0}^{\mu\left(\operatorname{co}\left(T \Omega_{n}\right)\right)} \varphi(t) d t\right) \\
& =\Psi\left(\int_{0}^{\mu\left(T \Omega_{n}\right)} \varphi(t) d t\right) \\
& \leq \Phi\left(\int_{0}^{\mu\left(\Omega_{n}\right)} \varphi(t) d t\right)
\end{aligned}
$$

hence we infer that $\left\{\int_{0}^{\mu\left(\Omega_{n}\right)} \varphi(t) d t\right\}$ is a decreasing sequence of positive real numbers by (1) of Definition 1.2. Thus there exists $r \geq 0$ such that both $\int_{0}^{\mu\left(\Omega_{n}\right)} \varphi(t) d t$ and $\int_{0}^{\mu\left(\Omega_{n+1}\right)} \varphi(t) d t$ converge to $r$ as $n \rightarrow \infty$. Then, in view of the above inequality and (2) of Definition 1.2, we get $r=0$ and hence

$$
\lim _{n \rightarrow \infty} \int_{0}^{\mu\left(\Omega_{n}\right)} \varphi(t) d t=0
$$

But for any $\varepsilon>0, \int_{0}^{\varepsilon} \varphi(t) d t>0$, thus $\mu\left(\Omega_{n}\right) \rightarrow 0$ as $n \rightarrow \infty$. 
Now since $\Omega_{n}$ is a nested sequence, in view of (6) of Definition 1.1, we conclude that $\Omega_{\infty}=\bigcap_{n=1}^{\infty} \Omega_{n}$ is a nonempty, closed and convex subset of $\Omega$. Moreover, we know that $\Omega_{\infty}$ belongs to ker $\mu$. So $\Omega_{\infty}$ is compact and invariant under the mapping $T$. Consequently, Theorem 1.4 implies that $T$ has a fixed point in $\Omega_{\infty}$. Since $\Omega_{\infty} \subset \Omega$, the proof is completed then.

Remark 2.2 Take $\varphi(t)=1$ for $t \in[0, \infty)$ in Theorem 2.1. Then

$$
\Psi\left(\int_{0}^{\mu(T X)} \varphi(t) d t\right)=\Psi(\mu(T X)) \leq \Phi(\mu(X))=\Phi\left(\int_{0}^{\mu(X)} \varphi(t) d t\right)
$$

then Theorem 6 of [4] is obtained.

Remark 2.3 Take

$$
\varphi(t)=1, \quad \Psi(t)=t, \quad \Phi(t)=k t \quad \text { for } t \in[0, \infty) \text { and } k \in[0,1)
$$

in Theorem 2.1. Then we have

$$
\mu(T X)=\Psi\left(\int_{0}^{\mu(T X)} \varphi(t) d t\right) \leq \Phi\left(\int_{0}^{\mu(X)} \varphi(t) d t\right)=k \mu(X),
$$

thus we get Darbo's fixed point theorem.

Remark 2.4 Set $\Psi(t)=t, t \in[0, \infty)$. Let the function $\Phi$ satisfy

$$
\lim _{n \rightarrow \infty} \Phi^{n}(t)=0 \quad \text { for any } t \geq 0
$$

Then we have

$$
\int_{0}^{\mu(T X)} \varphi(t) d t=\Psi\left(\int_{0}^{\mu(T X)} \varphi(t) d t\right) \leq \Phi\left(\int_{0}^{\mu(X)} \varphi(t) d t\right),
$$

thus we get Theorem 2.1 of [3].

Remark 2.5 It is easy to verify that $\Psi$ and $\Phi$ constructed above are a pair of shifting distance functions.

Corollary 2.6 Let $\Omega$ be a nonempty, closed, bounded and convex subset of a Banach space $E$ and let $T: \Omega \rightarrow \Omega$ be a mapping which satisfies the following inequality:

$$
\Psi\left(\int_{0}^{\|T x-T y\|} \varphi(t) d t\right) \leq \Phi\left(\int_{0}^{\|x-y\|} \varphi(t) d t\right) \quad \text { for each } x, y \in \Omega,
$$

where $\Psi, \Phi:[0,+\infty) \rightarrow R$ are a pair of shifting distance functions. Moreover, let $\varphi$ : $[0,+\infty) \rightarrow[0,+\infty]$ be a Lebesgue-integrable function, which is summable on each compact subset of $[0,+\infty)$ and

$$
\int_{0}^{\varepsilon} \varphi(t) d t>0 \quad \text { for each } \varepsilon>0
$$

Then $T$ has at least one fixed point in $\Omega$. 
Proof Define $\mu: \mathfrak{M}_{E} \rightarrow R_{+}, \mu(X)=\operatorname{diam}(X), X \in \mathfrak{M}_{E}$, where $\operatorname{diam}(X)$ is the diameter of the set $X$. It is easy to verify that $\mu$ is a measure of noncompactness on the space $E$. By assumption, we have

$$
\Psi\left(\int_{0}^{\sup _{x, y \in X}\|T x-T y\|} \varphi(t) d t\right) \leq \Phi\left(\int_{0}^{\sup _{x, y \in X}\|x-y\|} \varphi(t) d t\right),
$$

thus we get

$$
\Psi\left(\int_{0}^{\mu(T X)} \varphi(t) d t\right) \leq \Phi\left(\int_{0}^{\mu(X)} \varphi(t) d t\right)
$$

By Theorem 2.1 we get the result about $T$.

Now, motivated by the contractive condition in Theorem 3.1 of [7], we present another generalization of Darbo's fixed point theorem as follows.

Theorem 2.7 Let $\Omega$ be a nonempty, closed, bounded and convex subset of a Banach space $E$ and let $T: \Omega \rightarrow \Omega$ be a continuous mapping which satisfies the following inequality:

$$
\Psi\left(\int_{0}^{\mu(T X)} \varphi(t) d t\right) \leq \Psi\left(\int_{0}^{\mu(X)} \varphi(t) d t\right)-\Phi\left(\int_{0}^{\mu(X)} \varphi(t) d t\right)
$$

for any nonempty subset $X$ of $\Omega$, where $\mu$ is a measure of noncompactness and $\Psi, \Phi: R_{+} \rightarrow$ $R_{+}$are given functions such that $\Phi$ is lower semicontinuous and $\Psi$ is nondecreasing and continuous. Moreover, $\Phi(0)=0$ and $\Phi(t)>0$ for $t>0$, and $\varphi:[0,+\infty) \rightarrow[0,+\infty]$ is a Lebesgue-integrable function, which is summable on each compact subset of $[0,+\infty)$ and

$$
\int_{0}^{\varepsilon} \varphi(t) d t>0 \quad \text { for each } \varepsilon>0
$$

Then $T$ has at least one fixed point in $\Omega$.

Proof Consider the sequence $\left\{\Omega_{n}\right\}$ defined by

$$
\Omega_{0}=\Omega, \quad \Omega_{n}=\operatorname{co}\left(T \Omega_{n-1}\right) \quad \text { for } n \geq 0 .
$$

If there exists a natural number $n_{0}$ such that $\mu\left(\Omega_{n_{0}}\right)=0$, then $\Omega_{n_{0}}$ is compact. The Schauder fixed point theorem ensures that $T$ has a fixed point in $\Omega$. So, we assume that $\mu\left(\Omega_{n}\right)>0$ for $n=1,2,3, \ldots$. Note that

$$
\begin{aligned}
\Psi\left(\int_{0}^{\mu\left(\Omega_{n+1}\right)} \varphi(t) d t\right) & =\Psi\left(\int_{0}^{\mu\left(\operatorname{co}\left(T \Omega_{n}\right)\right)} \varphi(t) d t\right)=\Psi\left(\int_{0}^{\mu\left(T \Omega_{n}\right)} \varphi(t) d t\right) \\
& \leq \Psi\left(\int_{0}^{\mu\left(\Omega_{n}\right)} \varphi(t) d t\right)-\Phi\left(\int_{0}^{\mu\left(\Omega_{n}\right)} \varphi(t) d t\right) .
\end{aligned}
$$

Since $\Phi(t) \geq 0$ for any $t \in[0,+\infty)$. Then

$$
\Psi\left(\int_{0}^{\mu\left(\Omega_{n+1}\right)} \varphi(t) d t\right) \leq \Psi\left(\int_{0}^{\mu\left(\Omega_{n}\right)} \varphi(t) d t\right)
$$


Hence the positive sequence $\left\{\int_{0}^{\mu\left(\Omega_{n}\right)} \varphi(t) d t\right\}$ is decreasing, thus is convergent. Let

$$
\lim _{n \rightarrow \infty} \int_{0}^{\mu\left(\Omega_{n}\right)} \varphi(t) d t=r
$$

Take limit on both sides of the above inequality. Then

$$
\Psi(r) \leq \Psi(r)-\lim _{n \rightarrow \infty} \Phi\left(\int_{0}^{\mu\left(\Omega_{n}\right)} \varphi(t) d t\right)
$$

Thus

$$
\lim _{n \rightarrow \infty} \Phi\left(\int_{0}^{\mu\left(\Omega_{n}\right)} \varphi(t) d t\right)=0
$$

By the hypothesis of $\Phi$, we have

$$
\lim _{n \rightarrow \infty} \int_{0}^{\mu\left(\Omega_{n}\right)} \varphi(t) d t=0
$$

By a similar argument of Theorem 2.1, we get our result.

Remark 2.8 When $\varphi(t)=1$, we get Theorem 2.1 of [4].

Theorem 2.9 Let $\Omega$ be a nonempty, closed, bounded and convex subset of a Banach space $E$ and let $T: \Omega \rightarrow \Omega$ be a continuous mapping which satisfies the following inequality:

$$
\Psi\left(\int_{0}^{\mu(T X)} \varphi(t) d t\right) \leq \Phi\left(\int_{0}^{\mu(X)} \varphi(t) d t\right)-\theta\left(\int_{0}^{\mu(X)} \varphi(t) d t\right)
$$

for any nonempty subset $X$ of $\Omega$, where $\mu$ is a measure of noncompactness and $\Psi, \Phi, \theta$ : $R_{+} \rightarrow R_{+}$are three functions such that $\Phi$ and $\theta$ are bounded on any bounded interval in $[0,+\infty)$ and $\Psi$ is continuous. Moreover, assume that

(1) $\Psi(s) \leq \Phi(t) \Rightarrow s \leq t$

(2) $\theta(t)=0 \Leftrightarrow t=0$ and $\theta \geq 0$;

(3) for any sequence $\left\{s_{n}\right\}$ in $R_{+}$with $s_{n} \rightarrow t>0$,

$$
\Psi(t)-\lim _{n \rightarrow \infty} \sup \Phi\left(s_{n}\right)+\lim _{n \rightarrow \infty} \inf \theta\left(s_{n}\right)>0 .
$$

Proof Similar to the proof of Theorem 2.1, we have the same construction of the sequence $\left\{\Omega_{n}\right\}$. Without loss of generality, we assume $\mu\left(\Omega_{n}\right)>0$ for all $n \geq 0$. Considering the above inequality, we have

$$
\begin{aligned}
\Psi\left(\int_{0}^{\mu\left(\Omega_{n+1}\right)} \varphi(t) d t\right) & =\Psi\left(\int_{0}^{\mu\left(T \Omega_{n}\right)} \varphi(t) d t\right) \\
& \leq \Phi\left(\int_{0}^{\mu\left(\Omega_{n}\right)} \varphi(t) d t\right)-\theta\left(\int_{0}^{\mu\left(\Omega_{n}\right)} \varphi(t) d t\right) .
\end{aligned}
$$


Since $\theta \geq 0$, we see that

$$
\Psi\left(\int_{0}^{\mu\left(\Omega_{n+1}\right)} \varphi(t) d t\right) \leq \Phi\left(\int_{0}^{\mu\left(\Omega_{n}\right)} \varphi(t) d t\right)
$$

which implies

$$
\int_{0}^{\mu\left(\Omega_{n+1}\right)} \varphi(t) d t \leq \int_{0}^{\mu\left(\Omega_{n}\right)} \varphi(t) d t
$$

by condition (1). Thus $\left\{\int_{0}^{\mu\left(\Omega_{n}\right)} \varphi(t) d t\right\}$ is a positive but decreasing sequence. So there exists $r \geq 0$ such that

$$
\lim _{n \rightarrow \infty} \int_{0}^{\mu\left(\Omega_{n}\right)} \varphi(t) d t=r
$$

Therefore, by taking limit on both sides of the above inequality, we have

$$
\Psi(r) \leq \lim _{n \rightarrow \infty} \sup \Phi\left(\int_{0}^{\mu\left(\Omega_{n}\right)} \varphi(t) d t\right)+\lim _{n \rightarrow \infty} \sup -\theta\left(\int_{0}^{\mu\left(\Omega_{n}\right)} \varphi(t) d t\right) .
$$

Equivalently

$$
\Psi(r)-\lim _{n \rightarrow \infty} \sup \Phi\left(\int_{0}^{\mu\left(\Omega_{n}\right)} \varphi(t) d t\right)+\lim _{n \rightarrow \infty} \inf \theta\left(\int_{0}^{\mu\left(\Omega_{n}\right)} \varphi(t) d t\right) \leq 0
$$

Therefore,

$$
\lim _{n \rightarrow \infty} \int_{0}^{\mu\left(\Omega_{n}\right)} \varphi(t) d t=r=0
$$

and by the property of $\varphi$, we get

$$
\lim _{n \rightarrow \infty} \mu\left(\Omega_{n}\right)=0
$$

By the same discussion in Theorem 2.1, we see that the conclusion of the theorem is true.

Remark 2.10 When $\varphi(t)=1$, we get Theorem 9 of [6].

Remark 2.11 Taking

$$
\varphi(t)=1, \quad \Psi(t)=t, \quad \Phi(t)=t, \quad \theta(t)=(1-k) t, \quad t \in[0,+\infty), k \in[0,1),
$$

we get Darbo's fixed point theorem.

Let $\Psi(t)=t$ and $\theta(t)=0$ in the above theorem, we have the following corollary.

Corollary 2.12 Let $\Omega$ be a nonempty, closed, bounded and convex subset of a Banach space $E$ and let $T: \Omega \rightarrow \Omega$ be a continuous mapping which satisfies the following inequality:

$$
\int_{0}^{\mu(T X)} \varphi(t) d t \leq \Phi\left(\int_{0}^{\mu(X)} \varphi(t) d t\right)
$$


for any nonempty subset $X$ of $\Omega$, where $\mu$ is a measure of noncompactness and $\Phi: R_{+} \rightarrow R_{+}$ is bounded on any bounded interval in $[0,+\infty)$. Moreover, assume that $x \leq \Phi(y)$ implies $x \leq y$ and, for any sequence $\left\{s_{n}\right\}$ in $[0,+\infty)$ with $s_{n} \rightarrow t>0$,

$$
\lim _{n \rightarrow \infty} \sup \Phi\left(s_{n}\right)<t
$$

\section{Then Thas a fixed point.}

\section{Competing interests}

The authors declare that they have no competing interests.

\section{Authors' contributions}

Both authors contributed equally and significantly in writing this paper. Both authors read and approved the final manuscript.

\section{Acknowledgements}

The authors acknowledge support from NSFC (No. 11171210).

Received: 17 May 2015 Accepted: 13 August 2015 Published online: 28 August 2015

\section{References}

1. Darbo, G: Punti unitti in transformazioni a condominio non compatto. Rend. Semin. Mat. Univ. Padova 24, 84-92 (1955)

2. Kuratowski, K: Sur les espaces complets. Fundam. Math. 5, 301-309 (1930)

3. Aghajani, A, Allahyari, R, Mursaleen, M: A generalization of Darbo's theorem with application to the solvability of systems of integral equations. J. Comput. Appl. Math. 260, 68-77 (2014)

4. Aghajani, A, Banas, J, Sabzali, N: Some generalizations of Darbo fixed point theorem and applications. Bull. Belg. Math. Soc. Simon Stevin 20, 345-358 (2013)

5. Hajji, A: A generalization of Darbo's fixed point and common solutions of equations in Banach spaces. Fixed Point Theory Appl. 2013, 62 (2013)

6. Samadi, A, Ghaemi, MB: An extension of Darbo's theorem and its application. Abstr. Appl. Anal. 2014, Article ID 852324 (2014)

7. Choudhury, BS, Metiya, N, Postolache, M: A generalized weak contraction principle with applications to coupled coincidence point problems. Fixed Point Theory Appl. 2013, 152 (2013)

\section{Submit your manuscript to a SpringerOpen ${ }^{\odot}$ journal and benefit from:}

- Convenient online submission

Rigorous peer review

- Immediate publication on acceptance

- Open access: articles freely available online

- High visibility within the field

- Retaining the copyright to your article 